\title{
Complete Clinical Response in Locally Advanced Metastatic de novo Breast Cancer after Front-Line Treatment with Ribociclib/Letrozole within the RIBANNA Study
}

\author{
Christian Rudlowski Nina Beermann Lena Leitzen Benno Nuding \\ Breast Unit, Evangelisches Krankenhaus, Bergisch Gladbach, Germany
}

\section{Established Facts}

- Endocrine therapy is recommended for advanced hormone receptor-positive breast cancer.

- CDK 4/6 inhibitors significantly improve disease-free survival in human epidermal growth factor receptor 2-negative, hormone receptor-positive advanced breast cancer.

\section{Novel Insights}

- Complete clinical remission in a patient with locally advanced hormone receptor-positive, human epidermal growth factor receptor 2-negative de novo breast cancer treated with ribociclib + letrozole.

- Long-lasting remission of locally advanced and pulmonary metastatic breast cancer treated with ribociclib/letrozole within the RIBANNA trial.

\section{Keywords}

Complete clinical response - Locally advanced metastatic de novo breast cancer $\cdot$ Front-line treatment $\cdot$ Ribociclib . Letrozole

\footnotetext{
Abstract

Introduction: Ribociclib is an orally bioavailable cyclin-dependent kinase 4/6 inhibitor. In combination with aromatase inhibitor letrozole, it has approval for treatment of hormone receptor positive ( $\mathrm{HR}+$ ) and human epidermal growth factor receptor 2-negative (HER2-) advanced breast cancer. First-line therapy with ribociclib + letrozole significantly improves progression-free survival compared to placebo + letrozole in patients with $\mathrm{HR}+/ \mathrm{HER} 2-$ advanced breast cancer. In patients with de novo advanced or metastatic breast can-
}

cer, ribociclib was able to provide substantial clinical benefit according to data from the MONALEESA-2 study. Case Presentation: Here, we report the complete clinical response in a postmenopausal patient with de novo, locally advanced, pulmonary metastatic breast cancer treated with ribociclib + letrozole. Our patient presented an ulcerated breast-consuming tumor with multiple pulmonary metastases. $\mathrm{HR}+$ / HER2- breast cancer was confirmed by tumor biopsy. Ki67 expression was $90 \%$. After three months of initial treatment, the tumor-associated ulcerations disappeared, and no measurable pulmonary disease was detectable on CT scan. Treatment was well tolerated, and after dosage reduction due to neutropenia, no further side effects have been documented. At present, complete clinical response remains after 15 months of ongoing treatment. Conclusion: This case report documents an exceptional tumor response of a fast growing, 
locally advanced, pulmonary metastatic HR+/HER2- de novo breast cancer treated by ribociclib/letrozole combination therapy. Treatment success was long lasting with few side effects. The patient was very satisfied with the treatment and had no specific restrictions in her daily life.

(C) 2019 S. Karger AG, Basel

\section{Introduction}

A total of 3-25\% of all newly diagnosed patients present with de novo hormone receptor-positive $(\mathrm{HR}+)$ advanced breast cancer [1]. Current guidelines recommend to use first-line endocrine therapy, with or without a cyclin-dependent kinase (CDK) 4/6 inhibitor, in patients with de novo or relapsed $\mathrm{HR}+$ advanced breast cancer [2]. Three different CDK 4/6 inhibitors are currently available: palbociclib, ribociclib, and abemaciclib [3].

Ribociclib is an orally bioavailable CDK 4/6 inhibitor that has been approved for use in combination with aromatase inhibitor letrozole for the treatment of $\mathrm{HR}+/ \mathrm{hu}-$ man epidermal growth factor receptor 2-negative (HER2-) advanced breast cancer [4]. This approval is based on findings from the MONALEESA-2 study, a double-blind, placebo-controlled, randomized phase 3 trial (NCT01958021). First-line therapy with ribociclib + letrozole significantly improved progression-free survival compared with placebo + letrozole in patients with $\mathrm{HR}+$ / HER2 - advanced breast cancer [5]. Within the MONALEESA-2 study, only two patients with de novo advanced breast cancer receiving ribociclib + letrozole had complete clinical response (CCR) [6].

RIBANNA is an ongoing non-interventional study running in Germany since October 2017 [7]. 3,020 pre-/ postmenopausal patients are expected to be finally enrolled receiving ribociclib + aromatase inhibitors (AI), endocrine monotherapy, or chemotherapy as first-line treatment for HR+/HER2- advanced breast cancer. The aim of this study is to assess the safety and efficacy of different treatment options. Here, we report about a patient treated within the RIBANNA study with locally extended ulcerated breast cancer and multiple pulmonary metastases. She had had no prior systemic therapy and was treated with ribociclib + AI. After four cycles of treatment, CCR was achieved.

\section{Case Report}

A 60-year-old Caucasian female was referred to our institution with an enlarged and ulcerated lump in her left breast. She had had no prior history of breast cancer. Except mild hypertension, no preexisting conditions were reported. Clinical examination revealed an enormous ulcerated tumor in her left breast with widespread erythema and intense local edema (Fig. 1a). Pathological lymph node
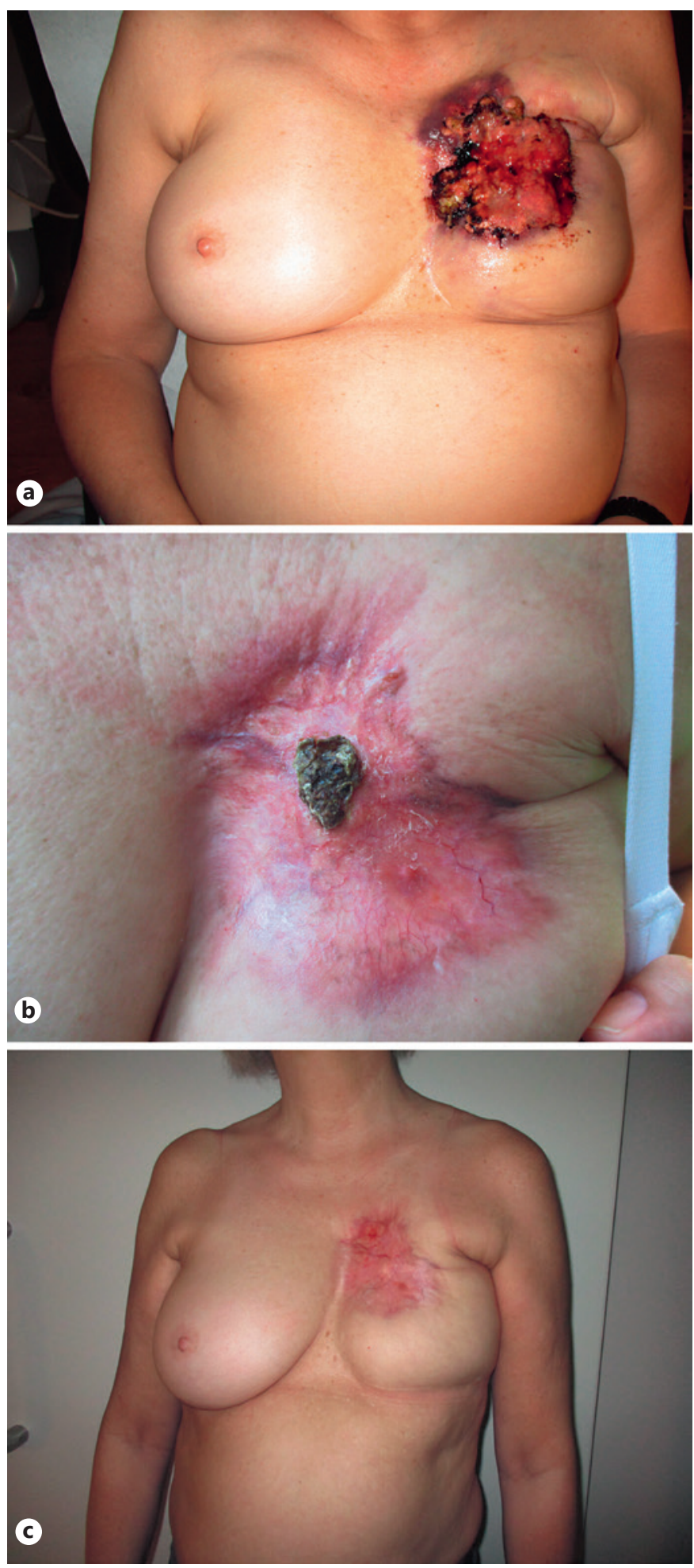

Fig. 1. Locally advanced breast cancer before (a) and after 4 (b) and 8 (c) cycles into the treatment.

involvement was palpable in the left axilla. Her right breast and regional lymph nodes showed no tumor involvement. Full body CT scan documented locally advanced breast disease with axillary lymph node involvement and both-sided pulmonary metastases (Fig. 2a). No other secondary deposits were observed. Scintigraphy showed no bone metastasis at that time. Tru-cut biopsies of the left breast de- 

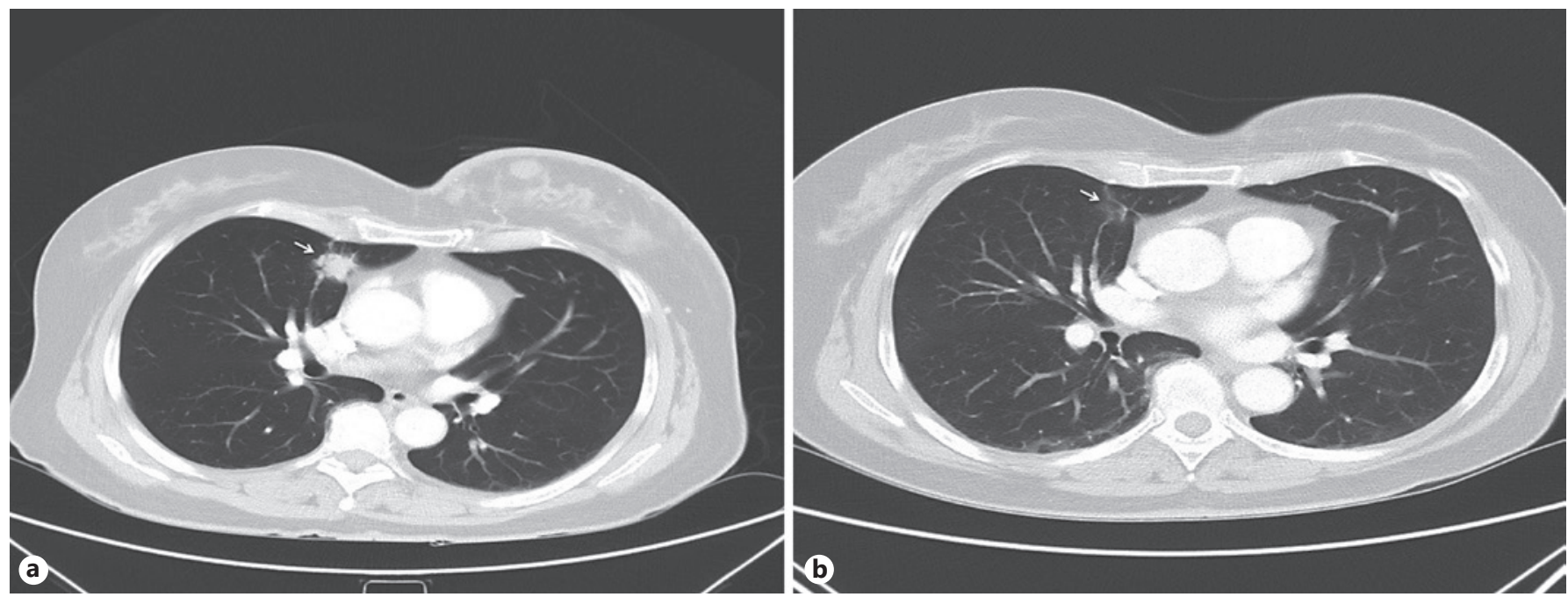

Fig. 2. CT scan of the chest before (a) and after 4 cycles into the treatment (b). Pulmonary metastasis in the left upper lobe is marked with a white arrow.

Table 1. Response rates of different CDK $4 / 6$ inhibitors in prospective randomized trials

\begin{tabular}{|c|c|c|c|c|c|c|}
\hline \multirow[t]{2}{*}{ Trial } & \multirow{2}{*}{$\begin{array}{l}\text { CDK } 4 / 6 \text { inhibitors + } \\
\text { endocrine treatment }\end{array}$} & \multirow{2}{*}{$\begin{array}{l}\text { Endocrine } \\
\text { treatment }\end{array}$} & \multicolumn{2}{|l|}{ CCR } & \multicolumn{2}{|l|}{ OR } \\
\hline & & & $\begin{array}{l}\text { CDK 4/6 } \\
\text { inhibitors }\end{array}$ & Control & $\begin{array}{l}\text { CDK 4/6 } \\
\text { inhibitors }\end{array}$ & Control \\
\hline MONALEESA-2 [9] & Ribociclib/Letrozol & Letrozol & $2.7 \%$ & $2.1 \%$ & $40.7 \%$ & $27.5 \%$ \\
\hline \multicolumn{7}{|l|}{ MONALEESA-2 } \\
\hline (de novo) [6] & Ribociclib/Letrozol & Letrozol & $2.0 \%$ & $1.0 \%$ & $47.0 \%$ & $34.0 \%$ \\
\hline MONALEESA-3 [8] & Ribociclib/Fulvestrant & Fulvestrant & $1.7 \%$ & $0 \%$ & $32.4 \%$ & $21.5 \%$ \\
\hline MONALEESA-7 [12] & Ribociclib/NSAI or Tam & NSAI or Tam & $2.0 \%$ & $2.0 \%$ & $41.0 \%$ & $30.0 \%$ \\
\hline PALOMA-1 [13] & Palbociclib/Letrozol & Letrozol & - & - & $42.1 \%$ & $34.7 \%$ \\
\hline PALOMA-3 [14] & Palbociclib/Fulvestrant & Fulvestrant & $0 \%$ & $2.0 \%$ & $19.0 \%$ & $9.0 \%$ \\
\hline \multicolumn{7}{|l|}{ PALLET (neoadj.) } \\
\hline$[15,16]$ & Palbociclib/Letrozol & Letrozol & $1.6 \%$ & $2.2 \%$ & $54.3 \%$ & $49.5 \%$ \\
\hline MONARCH 3 [16] & Abemaciclib/NSAI & NSAI & $1.5 \%$ & $0 \%$ & $48.2 \%$ & $34.5 \%$ \\
\hline MONARCH 2 [17] & Abemaciclib/Fulvestrant & Fulvestrant & $3.1 \%$ & $0.4 \%$ & $35.2 \%$ & $16.1 \%$ \\
\hline
\end{tabular}

CCR, complete clinical response; OR, overall response; NSAI, non-steroid aromatase inhibitor.

tected a ductal invasive carcinoma, G2, highly progesterone and estrogen receptor positive, HER2-. Ki67 was strongly expressed in more than $90 \%$ of the tumor cells. Our local tumor board recommended treatment with CDK 4/6 inhibitor ribociclib and letrozole.

After obtaining informed consent, the patient was included in the RIBANNA study - a non-interventional study running in Germany since October 2017 [7]. Standard blood tests including magnesium levels and electrocardiogram showed no pathological findings. On April 2, 2018, $600 \mathrm{mg}$ ribociclib + $2.5 \mathrm{mg}$ letrozole were prescribed. Following the manufactures' instructions and RIBANNA design, ribociclib was given daily for 3 weeks within a 4-week cycle in combination with letrozole continuously. During the first cycle, treatment was excellently tolerated, and no side effects were documented. After 3 weeks, before beginning the next cycle, our patient suffered from severe neutropenia $(<500 / \mu \mathrm{L})$. According to the RIBANNA protocol, treatment was stopped for 2 weeks, then the dosage was reduced to $400 \mathrm{mg}$ ribociclib daily. Before beginning the second cycle, no persisting neutropenia was documented.
During the following cycles, no further hematologic adverse event was observed. Beside mild persistent arthralgia and myalgia, no side effects were documented. Per protocol, the patient was routinely asked to fill in standardized questionnaires.

No further side effects were observed during the following treatment. Regular blood tests and electrocardiogram were normal. Clinical examination showed treatment response after two cycles. After four cycles, CCR could be verified in the breast and chest wall (Fig. 1b). CT scan confirmed CCR of pulmonary metastases (Fig. 2b). Treatment has been continued, and, at present, 16 cycles have been given without dosage reduction or treatment delay. After 15 months of treatment, tolerability is excellent, and no further side effects have occurred. Complete remission persists (Fig. 1c), and new tumor sites could not be detected by carefully clinical examination. Following the study protocol, treatment will be continued until tumor progression or treatment interruption due to severe toxicities. Treatment has exclusively been conducted in an outpatient fashion, and no hospital stay was required. 


\section{Discussion}

The large prospective randomized trial MONALEESA-2 has demonstrated that postmenopausal women with HR+/HER2- advanced breast cancer, treated with a combination of ribociclib/letrozole, had prolonged progression-free survival compared to those treated with letrozole alone $[8,9]$. Here, we present a patient with de novo metastatic HR+/HER2- breast cancer suffering from multiple pulmonary metastases and locally advanced ulcerated breast tumor. CCR was achieved after 4 cycles of ribociclib/letrozole. Treatment was well tolerated. After a dose reduction, as a result of neutropenia, only grade I adverse events were observed. These findings are of high interest due to a lack of data describing CCR of de novo breast cancer treated with CDK 4/6 inhibitors in combination with endocrine therapy $[6,10,11]$.

Actually, three different CDK 4/6 inhibitors are approved for treatment of locally advanced and/or metastatic HR+/HER2- breast cancer. Palbocilib, ribociclib, and abemaciclib showed significant improvement in the overall response rates compared to standard endocrine treatment. Data from prospective randomized trials (Table 1) demonstrated CCR rates from 0 to $3.1 \%$ depending on different CDK 4/6 inhibitors [6, 8, 9, 12-17]. However, due to the very low CCR rates in this particular subset of patients, we can only speculate about potential differences between the CDK 4/6 inhibitors.

In a subset of the MONALEESA-2 trial, CCR was observed in only 2 of 114 patients with de novo advanced breast cancer [6]. No data are available regarding specific tumor sites and local tumor growth in these two patients. Neoadjuvant treatment of primary HR+/HER2- breast cancer with CDK 4/6 inhibitor palbociclib in combination with letrozole, within the prospective randomized PALLET trial, did not increase the clinical response rate compared to letrozole alone [15]. In patients treated with palbociclib/letrozole, no pathological complete response was observed. However, malignant cell proliferation measured by Ki67 expression analyses revealed significant suppression after adding palbociclib to letrozole treatment in the neoadjuvant setting [15]. In our patient, Ki67 expression was 90\% before treatment, representing a fast-growing tumor burden. Due to CCR, no Ki67 expression after treatment was evaluable. We can only speculate that aggressive tumor growth measured by Ki67 is a predictor for clinical response in ribociclib treatment. In the PALLET trial, most of the patients had Ki67 values of $20 \%$ and below at the time of diagnosis. This might explain the excellent clinical response in the case reported here. In slower growing tumors, anti-proliferative effects will yield a slower reduction of tumor size [15]. Several trials with CDK 4/6 inhibitors demonstrated that antiproliferative effects of treatment correlate with improved disease-free survival [18]. When treatment is stopped, these effects diminish. In our patient, after 15 months of ongoing treatment, no tumor relapse has been observed.

In conclusion, the case reported here documents exceptional tumor response of a fast-growing locally advanced and pulmonary metastatic HR+/HER2- de novo breast cancer treated by ribociclib/letrozole combination therapy. Treatment success was long-lasting with few side effects. The patient is very satisfied with treatment and has no specific restrictions in her daily life.

\section{Acknowledgement}

The authors thanks Mrs. Lubig, M.D., Mrs. Neuenhaus, and Mrs. Feldmann-Pröpper for their technical and medical support.

\section{Statement of Ethics}

This work was conducted ethically in accordance with the World Medical Association Declaration of Helsinki. The patient has given her written informed consent to publish this case (including publication of images).

\section{Disclosure Statement}

The authors have no conflicts of interest to declare. C.R., L.L., and B.N. received honoraria for lectures and from advisory boards: C.R. from Roche Pharma, Astra Zeneca, and Novartis; L.L. from Novartis; and B.N. from Novartis.

\section{Funding Sources}

No funding has to declared.

\section{Author Contributions}

C.R. - Data collection and manuscript preparation.

N.B. - Data collection and paper revision.

L.L. - Data collection and paper revision.

B.N. - Data collection and paper revision.

References

1 Miao H, Hartman M, Bhoo-Pathy N, Lee SC, Taib NA, Tan EY, et al. Predicting survival of de novo metastatic breast cancer in Asian women: systematic review and validation study. PLoS One. 2014 Apr; 9(4):e93755.

2 Hortobagyi GN. Ribociclib for the first-line treatment of advanced hormone receptorpositive breast cancer: a review of subgroup analyses from the MONALEESA-2 trial. Breast Cancer Res. 2018 Oct;20(1):123. 
3 Spring L, Bardia A, Modi S. Targeting the cyclin D-cyclin-dependent kinase (CDK) 4/6-retinoblastoma pathway with selective CDK 4/6 inhibitors in hormone receptorpositive breast cancer: rationale, current status, and future directions. Discov Med. 2016 Jan;21(113):65-74.

4 Hortobagyi GN, Stemmer SM, Burris HA, Yap YS, Sonke GS, Paluch-Shimon S, et al. Ribociclib as first-line therapy for HR-positive, advanced breast cancer. N Engl J Med. 2016 Nov;375(18):1738-48.

5 Hortobagyi GN. Ribociclib for HR-positive, advanced breast cancer. N Engl J Med. 2017 Jan;376(3):289.

6 O’Shaughnessy J, Petrakova K, Sonke GS, Conte P, Arteaga CL, Cameron DA, et al. Ribociclib plus letrozole versus letrozole alone in patients with de novo HR+, HER2- advanced breast cancer in the randomized MONALEESA-2 trial. Breast Cancer Res Treat. 2018 Feb;168(1):127-34.

7 Fasching PA, Decker T, Guderian G, Heim J, Jakisch C, Jueck H, et al. Real-world effectiveness of ribociclib + aromatase inhibitor, or endocrine monotherapy, or chemotherapy as first-line treatment in postmenopausal women. Ann Oncol. 2018;29(suppl 8):viii87-9.

8 Slamon DJ, Neven P, Chia S, Fasching PA, De Laurentiis M, Im SA, et al. Phase III Randomized Study of Ribociclib and Fulvestrant in Hormone Receptor-Positive, Human Epidermal Growth Factor Receptor 2-Negative Advanced Breast Cancer: MONALEESA-3. J Clin Oncol. 2018 Aug;36(24):2465-72.
9 Hortobagyi GN, Stemmer SM, Burris HA, Yap YS, Sonke GS, Paluch-Shimon S, et al. Updated results from MONALEESA-2, a phase III trial of first-line ribociclib plus letrozole versus placebo plus letrozole in hormone receptor-positive, HER2-negative advanced breast cancer. Ann Oncol. 2018 Jul;29(7): 1541-7.

10 Ma CX, Gao F, Luo J, Northfelt DW, Goetz M, Forero A, et al. NeoPalAna: Neoadjuvant Palbociclib, a Cyclin-Dependent Kinase 4/6 Inhibitor, and Anastrozole for Clinical Stage 2 or 3 Estrogen Receptor-Positive Breast Cancer. Clin Cancer Res. 2017 Aug;23(15):405565.

11 Cottu P, D’Hondt V, Dureau S, Lerebours F, Desmoulins I, Heudel PE, et al. Letrozole and palbociclib versus chemotherapy as neoadjuvant therapy of high-risk luminal breast cancer. Ann Oncol. 2018 Dec;29(12):2334-40.

12 Tripathy D, Im SA, Colleoni M, Franke F, Bardia A, Harbeck N, et al. Ribociclib plus endocrine therapy for premenopausal women with hormone-receptor-positive, advanced breast cancer (MONALEESA-7): a randomised phase 3 trial. Lancet Oncol. 2018 Jul; 19(7):904-15.

13 Finn RS, Crown JP, Ettl J, Schmidt M, Bondarenko IM, Lang I, et al. Efficacy and safety of palbociclib in combination with letrozole as first-line treatment of ER-positive, HER2negative, advanced breast cancer: expanded analyses of subgroups from the randomized pivotal trial PALOMA-1/TRIO-18. Breast Cancer Res. 2016 Jun;18(1):67.
14 Cristofanilli M, Turner NC, Bondarenko I, Ro J, Im SA, Masuda N, et al. Fulvestrant plus palbociclib versus fulvestrant plus placebo for treatment of hormone-receptor-positive, HER2-negative metastatic breast cancer that progressed on previous endocrine therapy (PALOMA-3): final analysis of the multicentre, double-blind, phase 3 randomised controlled trial. Lancet Oncol. 2016 Apr;17(4): 425-39.

15 Johnston S, Puhalla S, Wheatley D, Ring A, Barry P, Holcombe C, et al. Randomized Phase II Study Evaluating Palbociclib in Addition to Letrozole as Neoadjuvant Therapy in Estrogen Receptor-Positive Early Breast Cancer: PALLET Trial. J Clin Oncol. 2019 Jan;37(3):178-189.

16 Goetz MP, Toi M, Campone M, Sohn J, Paluch-Shimon S, Huober J, et al. MONARCH 3: Abemaciclib As Initial Therapy for Advanced Breast Cancer. J Clin Oncol. 2017 Nov;35(32):3638-46.

17 Sledge GW Jr, Toi M, Neven P, Sohn J, Inoue K, Pivot X, et al. MONARCH 2: Abemaciclib in Combination With Fulvestrant in Women With HR+/HER2- Advanced Breast Cancer Who Had Progressed While Receiving Endocrine Therapy. J Clin Oncol. 2017 Sep;35(25): 2875-84.

18 Kwapisz D. Cyclin-dependent kinase 4/6 inhibitors in hormone receptor-positive early breast cancer: preliminary results and ongoing studies. Breast Cancer. 2018 Sep;25(5): 506-16. 